\title{
Hydrodynamic Deformation and Removal of Staphylococcus epidermidis Biofilms Treated With Urea, Chlorhexidine, Iron Chloride, or DispersinB
}

\author{
Eric R. Brindle, ${ }^{1}$ David A. Miller, ${ }^{1}$ Philip S. Stewart ${ }^{2}$ \\ ${ }^{l}$ Department of Mechanical and Industrial Engineering, Montana State University, \\ 220 Roberts Hall, Bozeman, Montana 59717-1800; telephone: (406) 994-2203; \\ fax: (406) 994-6292; e-mail: dmiller@me.montana.edu \\ ${ }^{2}$ Center for Biofilm Engineering, Montana State University, Bozeman, Montana
}

Received 1 December 2010; revision received 12 June 2011; accepted 14 June 2011

Published online 5 July 2011 in Wiley Online Library (wileyonlinelibrary.com). DOI 10.1002/bit.23245

\begin{abstract}
The force-deflection and removal characteristics of bacterial biofilm were measured by two different techniques before and after chemical, or enzymatic, treatment. The first technique involved time lapse imaging of a biofilm grown in a capillary flow cell and subjected to a brief shear stress challenge imparted through increased fluid flow. Biofilm removal was determined by calculating the reduction in biofilm area from quantitative analysis of transmission images. The second technique was based on microindentation using an atomic force microscope. In both cases, biofilms formed by Staphylococcus epidermidis were exposed to buffer (untreated control), urea, chlorhexidine, iron chloride, or DispersinB. In control experiments, the biofilm exhibited force-deflection responses that were similar before and after the same treatment. The biofilm structure was stable during the post-treatment shear challenge ( $1 \%$ loss). Biofilms treated with chlorhexidine became less deformable after treatment and no increase in biomass removal was seen during the post-treatment shear challenge ( $2 \%$ loss). In contrast, biofilms treated with urea or DispersinB became more deformable and exhibited significant biofilm loss during the post-treatment flow challenge ( $71 \%$ and $40 \%$, respectively). During the treatment soak phase, biofilms exposed to urea swelled. Biofilms exposed to iron chloride showed little difference from the control other than slight contraction during the treatment soak. These observations suggest the following interpretations: (1) chemical or enzymatic treatments, including those that are not frankly antimicrobial, can alter the cohesion of bacterial biofilm; (2) biocidal treatments (e.g., chlorhexidine) do not necessarily weaken the biofilm; and (3) biofilm removal following treatment with agents that make the biofilm more deformable (e.g., urea, DispersinB) depend on interaction between the moving fluid and the biofilm structure. Measurements such as those reported here open the door to development of new technologies for controlling detrimental biofilms
\end{abstract}

Correspondence to: D.A. Miller

Additional Supporting Information may be found in the online version of this article. by targeting biofilm cohesion rather than killing microorganisms.

Biotechnol. Bioeng. 2011;108: 2968-2977.

(C) 2011 Wiley Periodicals, Inc.

KEYWORDS: biofilm; biofilm deformation; biofilm treatment; biofilm removal

\section{Introduction}

Microorganisms in biofilms produce extracellular polymeric substances (EPS) made up of hydrated polysaccharide, protein, and nucleic acid that protect them and provide mechanical stability (Flemming et al., 2000). It is the cohesiveness of the extracellular matrix that enables biofilms to accumulate to the extent that they cause pronounced pressure drop in industrial piping systems or increased drag on ship hulls (Townsin, 2003). When biofilms detach they cause other problems. Detachment can lead to transmission of pathogens in drinking water systems (Walker et al., 1995) or food processing facilities (Vieira et al., 1993). The tenacious adherence and cohesion of bacteria in biofilms likely contributes to the persistent infections that can develop on implanted medical devices (Costerton et al., 1999).

One of the purposes of studying the mechanical response of biofilm is to develop better ways to remove, or eradicate, detrimental biofilms. Mechanical removal is an effective biofilm control strategy, e.g., tooth brushing. For this example, removal of biofilm is achieved by shear forces applied through direct contact of toothbrush bristles on the gums and teeth (Vinogradov et al., 2004). Conventional biofilm control relies heavily on the use of biocides and antibiotics that kill microorganisms. This is partly due to the fact that much more is known about killing microorganisms with antimicrobials than is known about the chemistry and mechanics of biofilm dispersal and removal. 
To design more effective biofilm control approaches, it is important to know how chemical or enzymatic treatments alter the mechanical response of the biofilm and so change the ability to remove biofilm by application of a force. Relatively little work has been done to measure changes in biofilm mechanical response and removal as a result of such treatments. Stoodley et al. (2001) reported that two types of bacterial biofilm became much less susceptible to deformation by fluid flow after exposure to the trivalent aluminum ion. Similiarly, calcium concentration influenced the shear modulus and relaxation time of biofilms formed by a mucoid strain of Pseudomonas aeruginosa (Wloka et al., 2004). Other investigators report that the presence of added calcium or iron in the growth medium increases biofilm cohesiveness (Ahimou et al., 2007; Mohle et al., 2007). A recent investigation reported stiffening of $P$. aeruginosa biofilms by trivalent iron and aluminum and notable reduction in elasticity by citric acid (Lieleg et al., 2011). The stabilizing effect of multivalent cations is likely due to electrostatic cross-linking of negatively charged polymers in the EPS (Chen and Stewart, 2002).

The reality that antimicrobial treatments may not cause any biofilm removal was demonstrated visually in the work of Davison et al. (2010) who imaged biofilm in flow cells during exposure to a flowing solution of antimicrobial agent. Control, nisin, quaternary ammonium compound, and glutaraldehyde treatments did not result in any removal or deformation of the biofilm. Treatment with chlorine caused a steady erosion of biomass that was evidently dependent on hydrodynamic shear stress as it was more pronounced in the center of the flow cell where shear forces were higher. Sodium hydroxide treatment weakened biofilm and resulted in removal in a similar fashion in the same experimental system (Xavier et al., 2005). Simoes et al. (2005a, 2005b, 2009) treated biofilms with various biocides then measured biofilm removal resulting from increased fluid shear. They showed, for example, that treatment with glutaraldehyde or benzalkonium chloride (a quaternary ammonium compound) reduced the post-treatment removal by a mechanical challenge whereas chlorine and sodium hydroxide treatments increased post-treatment removal. Other investigation has shown that treatments that are not frankly antimicrobial, such as urea and sodium chloride, can nevertheless cause substantial biofilm removal (Chen and Stewart, 2000).

In focusing on biofilm removal it is important to recognize that removal represents cohesive material failure, for which an appropriate material property is an ultimate strength (Aggarwal and Hozalski, 2010; Aggarwal et al., 2010). A number of investigations have reported quantitative measurement of cohesive strengths (Aggarwal and Hozalski, 2010; Aggarwal et al., 2010; Chen et al., 2005; Mohle et al., 2007; Ohashi and Harada, 1994, 1996; Ohashi et al., 1999; Poppele and Hozalski, 2003). Measurements of other biofilm material properties such as elastic moduli or viscoelastic parameters may or may not correlate with biofilm removal, as these measurements pertain to deformation of the material but not failure.
The purpose of the work reported in this article is to describe and characterize the process of biofilm failure by direct microscopic visualization and to relate biofilm removal to measurements of biofilm deformation under hydrodynamic shear (in the flow cell) and compression (by microindentation), resulting from treatment with urea, chlorhexidine, iron chloride, and DispersinB.

\section{Materials and Methods}

\section{Treatments}

The treatments for this work were chosen based on effects on Staphylococcus epidermidis biofilm reported by Jones et al. (2011). The four treatments used in this research were urea, chlorhexidine, iron chloride, and DispersinB. Each of these treatments has been shown to affect the mechanical properties of the S. epidermidis biofilm through different mechanisms.

Urea is theorized to disrupt the hydrogen-bonding interactions thought to be important for EPS mechanical stability. Disrupting the hydrogen bonds should disrupt the EPS matrix resulting in a weaker biofilm (Chen and Stewart, $2000)$. Urea at a concentration of $30.0 \mathrm{~g} / \mathrm{L}(0.5 \mathrm{M})$ was used in this study. Chlorhexidine is a chemical antiseptic commonly used in low concentrations in contact lens solutions and in dental mouthwash. Chlorhexidine digluconate at a concentration of $1.0 \mathrm{~g} / \mathrm{L}(0.1 \%)$ was used in this study. Iron chloride $\left(\mathrm{FeCl}_{2}\right)$ has been shown to increase the apparent viscosity of certain biofilms (Chen and Stewart, 2002). When put into solution, iron chloride forms the cation $\mathrm{Fe}^{2+}$, which is capable of binding or cross-linking two negatively charged sites in the EPS. Iron chloride at a concentration of $12.7 \mathrm{~g} / \mathrm{L}(0.1 \mathrm{M})$ was used in this study. Dispersin B catalyzes the hydrolysis of poly- $N$-acetylglucosamine, a sticky extracellular polysaccharide produced by various bacteria including $S$. aureus and $S$. epidermidis (Kaplan et al., 2003). DispersinB has been shown to effectively disrupt $S$. epidermidis creating a softer, less consolidated biofilm cluster (Chaignon et al., 2007). DispersinB (provided by Kane Biotech, Inc., Winnipeg, MB Canada) at a concentration of $40 \mathrm{mg} / \mathrm{Lwas}$ used in this study.

\section{Capillary Flow Cell Methods}

Utilizing the flow cell technique, a version of the standard creep-recovery mechanical test is performed on the biofilm. A creep-recovery test involves applying a near instantaneous constant stress to a structure and measuring the time dependent deformation, or creep. The test is completed by removing the stress and measuring the time dependent recovery of the deformation toward the original configuration. The flow cell technique of the creep-recovery test utilizes the constant velocity of the flow cell medium to 
apply a hydrodynamic stress to the biofilm structure through the fluid-structure interaction.

An initial culture was started for inoculation using full strength tryptic soy broth (TSB) with S. epidermidis (ATCC 35984). The S. epidermidis inoculum incubated for $24 \mathrm{~h}$ on a shaker plate at $37^{\circ} \mathrm{C}$. The 24 -h culture was then used to inoculate a square glass capillary flow cell reactor of $0.9 \mathrm{~mm}$ ID and $170 \mu \mathrm{m}$ thick (Friedrich \& Dimmock, Millville, NJ) in which $2 \mathrm{~h}$ was allowed for attachment before the flow of nutrients was initiated. The reactor was then pumped with $10 \%$ TSB through the glass capillaries at a flow rate of $1 \mathrm{~mL} /$ min for $24 \mathrm{~h}$, at $37^{\circ} \mathrm{C}$.

The glass capillary reactor, which now contained the mature biofilm, was removed and viewed using a Nikon (Nikon Corp, Melville, NY) Eclipse E800 microscope, Hamamatsu (Hamamatsu Photonics, Bridgewater, NJ) C2400-75AH camera and MetaVue software. The spatial resolution of the microscope and camera was 0.97 microns/ pixel in both $x$ and $y$ coordinates. Using a Cole-Palmer continuous cycle syringe pump (R-74901-50) a $10 \mathrm{~mL} / \mathrm{min}$ (Reynolds number 230) shear test using 10\% TSB was applied to the biofilm for $15 \mathrm{~s}$ while recording one frame every $0.3 \mathrm{~s}$ for $30 \mathrm{~s}$ to capture both elongation and recovery of a particular biofilm cluster. This flow rate ensures laminar flow within the flow cell and represents a typical fluid shear challenge of a biofilm structure (Stoodley et al., 1999). This was repeated three times to create three trials of biofilm structure in the flow cell.

Figure 1 shows a collection of images taken during a capillary flow test. The measurements of biofilm deformation are seen in panels A-D. To analyze deformation, three reference points (red dots in panels B, C, and D) were identified on a cell cluster prior to flow challenge (B). The location of these points was marked at all times during the flow challenge $(\mathrm{C})$ using the MetaMorph digital imaging software. The vectors describing the change from $\mathrm{B}$ to $\mathrm{C}$ were calculated (D) and the magnitude of each vector recorded. Due to the 3-D nature of the biofilm structure growing in the flow cell and the hydrodynamic forces acting on the biofilm, the displacement vectors had small portions of deformation perpendicular to the flow direction. This deformation was considered in the analysis process by using the magnitude of the displacement vector. Using the timelapse images over the entire 30-s experiment, displacement vector magnitudes were measured throughout the hydrodynamic shear challenge and subsequent recovery. These three displacement vectors were normalized, then averaged at each time increment for the three trials, and plotted with respect to time (Fig. 2).

Recording biomass area throughout the initial shear test and the post-treatment shear test provides insights about the removal of the biofilm. The portion of the image identified as biomass was measured using the MetaVue software package, as shown in panels E-H of Figure 1. To analyze biofilm removal, the image was binary thresholded to define the biomass area prior to flow challenge (F) and also after flow challenge $(G)$. The biofilm area loss reported in Table I was calculated directly from the number of pixels thresholded. By subtracting the thresholded images in panels $F$ and $G$, a color-coded visualization $(\mathrm{H})$ was obtained showing where filled pixels have become empty (red) and empty pixels have become filled (green). This approach, employed in Figure 3, facilitates identification of detachment events and displacement of the cluster.

\section{Micro Indentation Methods}

An initial culture was started for inoculation using full strength TSB with S. epidermidis (ATCC 35984, ATCC Manassas, VA). The S. epidermidis inoculum incubated for $24 \mathrm{~h}$ on a shaker plate at $37^{\circ} \mathrm{C}$. The 24 -h culture was then used to inoculate a drip flow biofilm reactor (Bio Surface Technologies, Bozeman, MT) placed in a horizontal
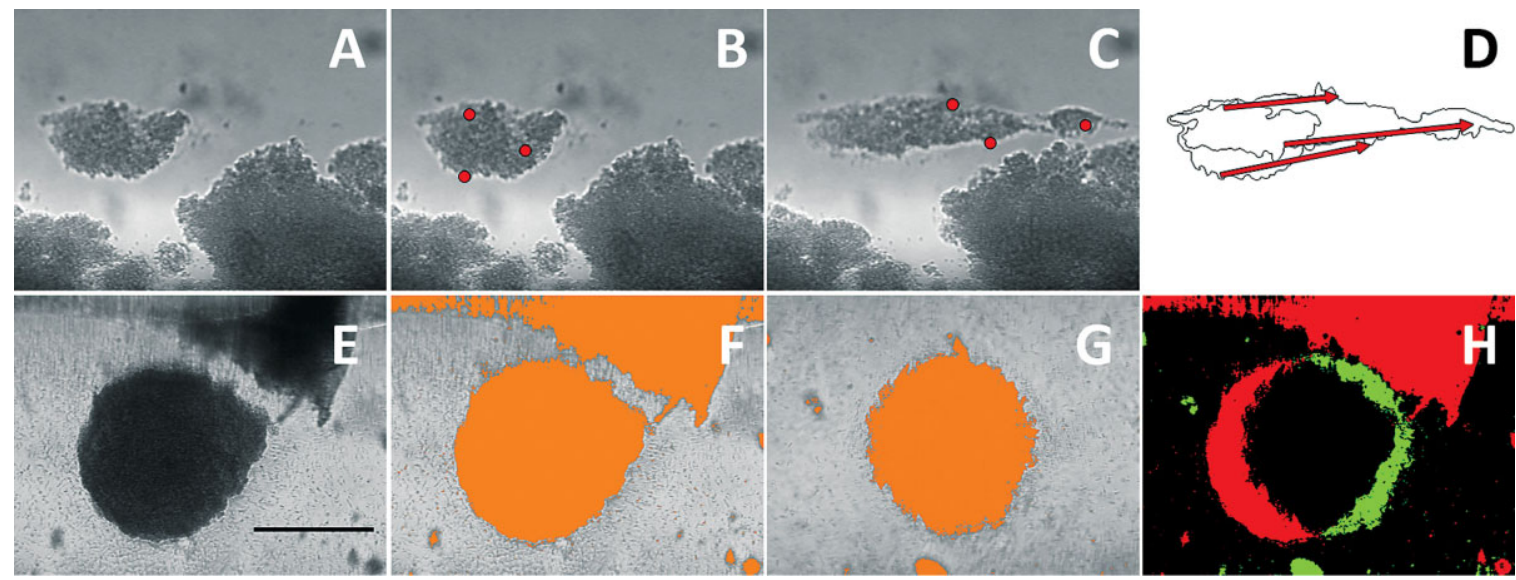

Figure 1. Illustration of measurement technique for deformation (A-D) and removal (E-H). The biofilm structure was imaged in transmission mode (A,E). Panels $A, B, E$, and $F$ were imaged prior to flow challenge whereas panels $\mathrm{C}$ and $\mathrm{G}$ were imaged during and after flow challenge, respectively. Flow was from left to right. 

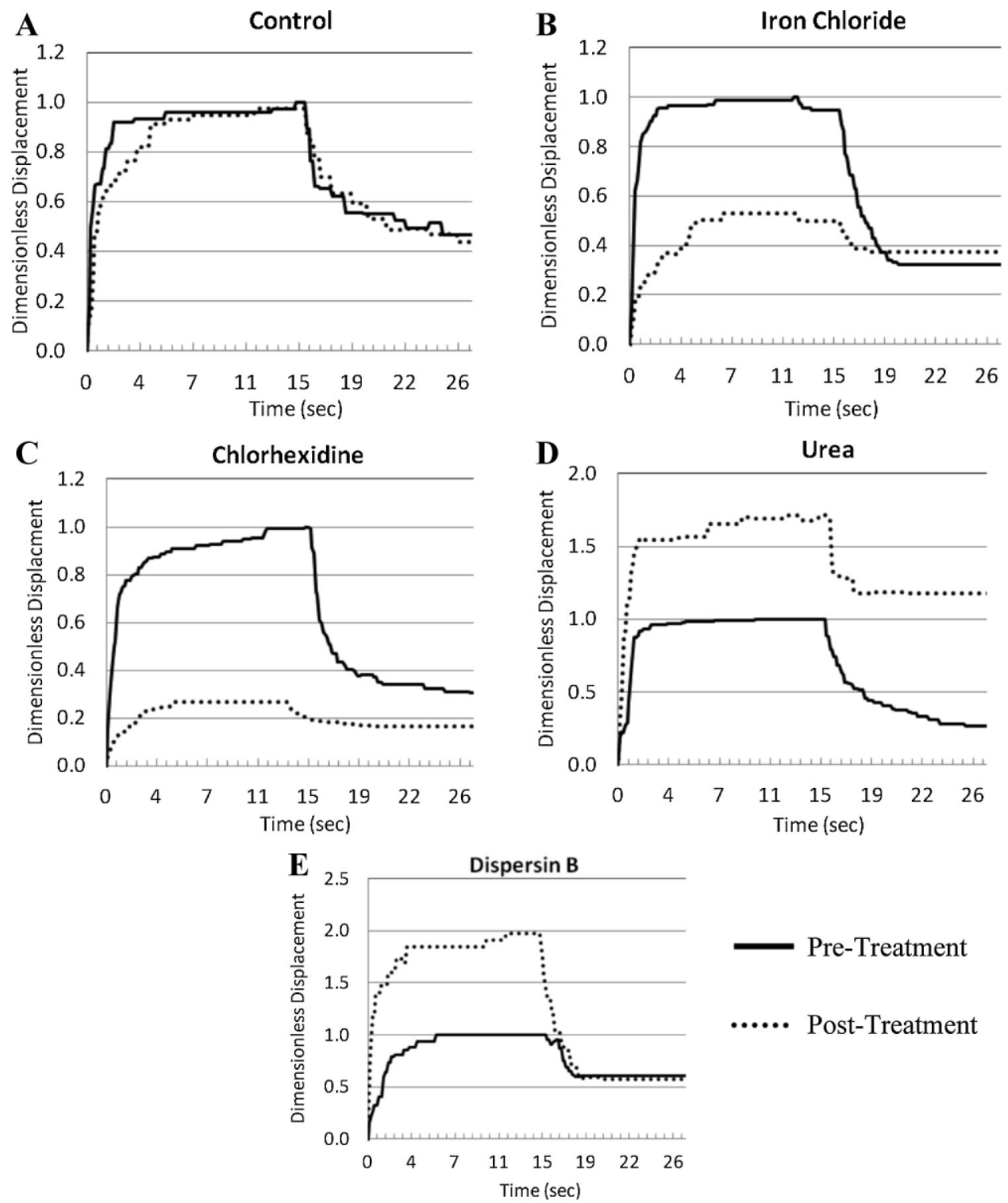

Pre-Treatment

...... Post-Treatment

Figure 2. Average captured creep-recovery displacements normalized to maximum displacement of the pre-treatment fluid shear trial from capillary flow cell experiments. All trials received a 15 min soak period with (A) control buffer solution, $(\mathbf{B}) \mathrm{FeCl}_{2}$ (C) chlorhexidine, (D) urea, and (E) DispersinB.

position with $1 \mathrm{~mL}$ of overnight culture and $9 \mathrm{~mL}$ of full strength TSB in each well. A small piece of autoclave tape was placed on the metal slide in the drip flow reactor to support a $1 \times 1 \mathrm{~cm}$ silicon wafer with a thin silicon dioxide surface. Two hours were allowed for attachment of cells, after which the inoculum bath was drained and the reactor was moved to an inclined position in which nutrient flow began. Nutrient flow consisted of $10 \%$ TSB dripped through each well at a flow rate of $1 \mathrm{~mL} / \mathrm{min}$ for $4 \mathrm{~h}$ in an incubator set to $37^{\circ} \mathrm{C}$. After a thin layer of biofilm had grown on the wafer it was removed and tested using a Veeco MultiMode PicoForce atomic force microscope in conjunction with diNanoScope (Veeco, Plainview, NY) Software version 7.0.

A force-displacement curve is generated by measuring the cantilever deflection during the loading and the unloading of the biofilm structure. The biofilm was tested using a $30 \mu \mathrm{m}$ diameter glass sphere indenter, which was mechanically cleaned prior to each biofilm and treatment experiment. Instrument controlled loading rates were utilized for each test at $1 \mu \mathrm{m} / \mathrm{s}, 10 \mu \mathrm{m} / \mathrm{s}$, and $100 \mu \mathrm{m} / \mathrm{s}$ to qualitatively identify rate effects of the biofilm. Additionally, displacement calibration of the AFM was verified prior to all testing. The cantilever stiffness was calibrated using the silicon wafer as a perfectly rigid object as compared to the stiffness of the cantilever beam. The measured cantilever stiffness was nominally $18 \mathrm{~N} / \mathrm{m}$.

After initial indentation, the biofilm was treated and allowed to soak for $15 \mathrm{~min}$. The same loading rates and indentation depths were then reapplied to the same point on the biofilm. Testing the identical spot before and after 
Table I. Percent recovery of deformation after unloading in flow cell experiments for pre-treatment and post-treatment conditions. ${ }^{*}-$ Failure recorded at $1\left(^{*}\right), 2\left(^{* *}\right)$, or $3(\mathrm{~F})$ points on the the biofilm.

\begin{tabular}{|c|c|c|c|c|}
\hline & & $\begin{array}{c}\% \text { Recovery } \\
\text { pre-treatment }\end{array}$ & $\begin{array}{c}\% \text { Recovery } \\
\text { post-treatment }\end{array}$ & $\begin{array}{c}\text { Max deformation ratio } \\
\text { (post-treatment/pre-treatment) }\end{array}$ \\
\hline \multirow[t]{4}{*}{ Control treatment } & Trial\#1 & 57.7 & 18.4 & 1.22 \\
\hline & Trial \#2 & 60.8 & 51.6 & 0.92 \\
\hline & Trial \#3 & 63.2 & 69.9 & 0.73 \\
\hline & Average $\pm \mathrm{SD}$ & $60.6 \pm 2.8$ & $46.6 \pm 26.1$ & $0.95 \pm 0.25$ \\
\hline \multirow[t]{4}{*}{ Iron chloride treatment } & Trial\#1 & 74.6 & 0.0 & 0.46 \\
\hline & Trial \#2 & $34.2^{*}$ & $22.9^{*}$ & 0.62 \\
\hline & Trial \#3 & 53.7 & 36.3 & 2.44 \\
\hline & Average $\pm \mathrm{SD}$ & $54.2 \pm 20.2$ & $19.7 \pm 18.3$ & $1.17 \pm 1.1$ \\
\hline \multirow[t]{4}{*}{ Chlorhexidine treatment } & Trial\#1 & 85.4 & 60.4 & 0.39 \\
\hline & Trial \#2 & 63.4 & 12.9 & 0.18 \\
\hline & Trial \#3 & 48.5 & 23.6 & 0.76 \\
\hline & Average \pm SD & $65.8 \pm 18.5$ & $32.3 \pm 24.9$ & $0.44 \pm 0.30$ \\
\hline \multirow[t]{4}{*}{ Urea treatment } & Trial\#1 & 68.5 & 10.5 & 2.09 \\
\hline & Trial \#2 & 54.0 & 70.9 & 4.69 \\
\hline & Trial \#3 & 69.8 & $\mathrm{~F}$ & $\mathrm{~F}$ \\
\hline & Average $\pm \mathrm{SD}$ & $64.1 \pm 8.8$ & $40.7 \pm 42.7$ & $3.39 \pm 1.83$ \\
\hline \multirow[t]{4}{*}{ DispersinB treatment } & Trial\#1 & 31.7 & $64.4^{* *}$ & 1.36 \\
\hline & Trial \#2 & 28.0 & $79.9^{* *}$ & 4.67 \\
\hline & Trial \#3 & 53.7 & $\mathrm{~F}$ & $\mathrm{~F}$ \\
\hline & Average $\pm \mathrm{SD}$ & $37.8 \pm 13.9$ & $72.2 \pm 11.0$ & $3.02 \pm 2.34$ \\
\hline
\end{tabular}

treatment is critical to comparing the effects of treatment on biofilm mechanical response. It is important to note that the biofilm grown on the wafer does not consist of a uniform material, but rather an inhomogeneous collection of biofilm clusters and geometries. Therefore, the location of the indent on the biofilm structure strongly influences the result, and testing the identical spot of the biofilm was identified to be the best method to determine the influence of treatment on the mechanical response. Tip cleanliness after indent was monitored by residual load of the cantilever. When the indenter returned to a zero load condition after removal from the biofilm, the indenter was identified as clean and ready for the next test. If a load existed on the cantilever, a tip cleaning procedure was employed. Three trials for each treatment and loading rate were conducted.

\section{Results}

\section{Capillary Flow Cell Results}

When a S. epidermidis biofilm grown in a capillary flow cell at a medium flow rate of $1 \mathrm{~mL} / \mathrm{min}$ was subjected to a 10 fold increase in flow rate, the biofilm deformed in the downstream direction (Fig. 1). When the flow was stopped, the biofilm returned partway to its original position. Videos of this experiment can be viewed in the Supporting Information.

By plotting the normalized and averaged displacement magnitudes of reference points on the biofilm during this type of experiment, typical creep-recovery curves were obtained, seen in Figure 2. The biofilm deformed in a reproducible manner in untreated biofilms (solid lines in all panels of Fig. 2). Table I indicates the percentage of recovery for pre-treatment and post-treatment tests for each trial of the various treatments. Table I also contains the number of failures of biofilm structure that occurred during the hydrodynamic testing. Failure is defined as the portion of the biofilm containing the reference point separating from the biofilm mass and travelling out of the frame.

The control for this protocol was to perform back-to-back creep tests on the same biofilm in which the $15 \mathrm{~min}$ treatment soak was performed with buffer solution rather than a treatment agent. In such repeated controls, the second creep test reproduced results of the first test (Fig. 2A). The percentage of recovery after the second stress test, Table I, was lower (47\%) than for the first test $(61 \%)$. This difference, however, was not statistically significant $(P=0.59)$. The maximum deformation of the biofilm in the second stress test was an average $95 \%$ of the maximum deformation in the first stress test (column 3 in Table I).

Table I and Figure 2 also show that the percent recovery after treatment is reduced for all treatments except DispersinB. DispersinB created seven failures of the biofilm during the post-treatment test. A statistical analysis of the displacements shown in Table I show that at $10 \%$ significance only the urea treatment is statistically different from the control.

Qualitative behaviors of biofilm structures in response to flow challenges and the various treatments are illustrated in Figure 3 for representative experiments. Each row of this figure summarizes the changes in structure and loss, or gain, of biomass area that occur in a single experiment. In the untreated control, the relative absence of color in panels B$\mathrm{D}$ indicates little net loss (red) or gain (green) of biomass in 
A

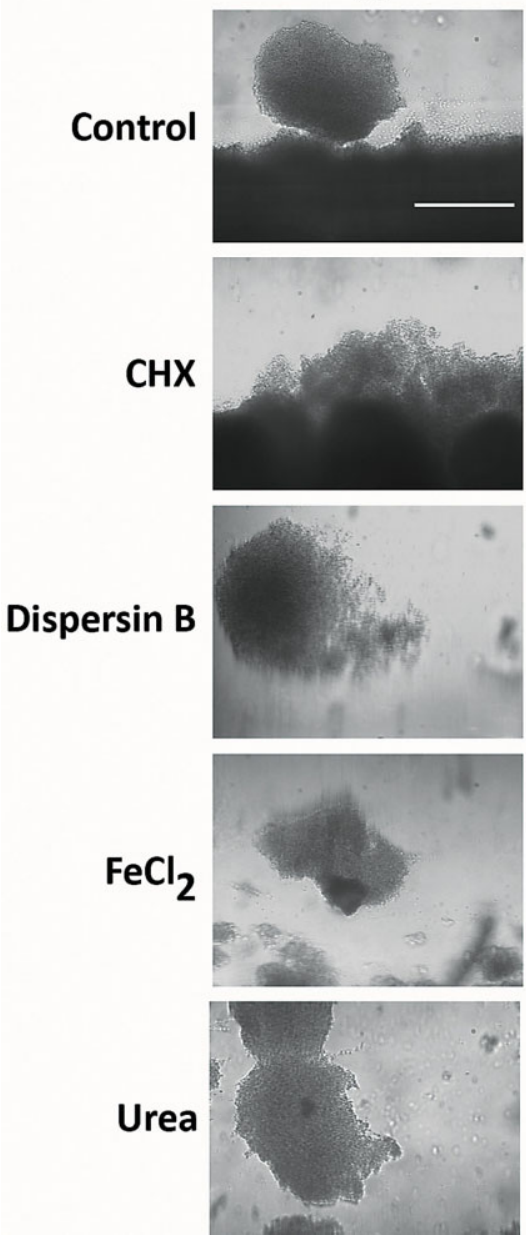

B
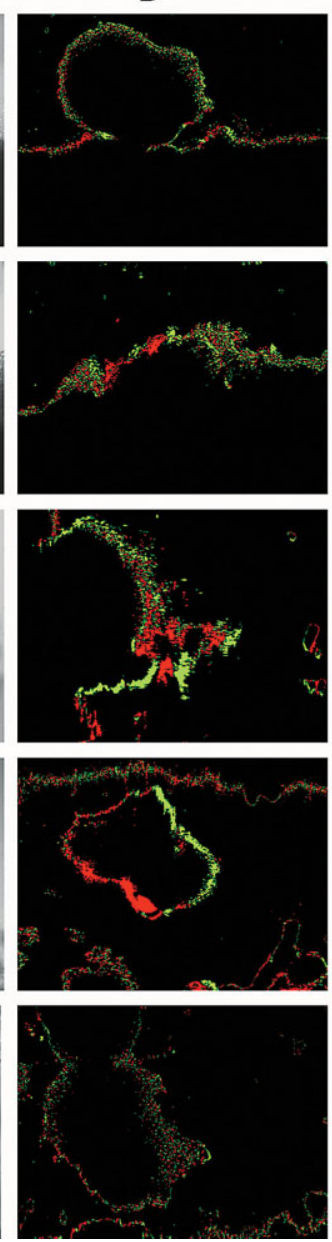
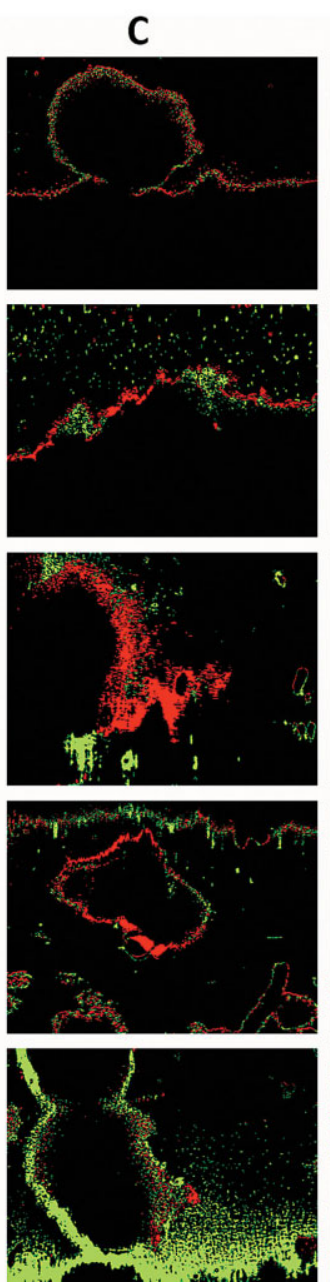

D
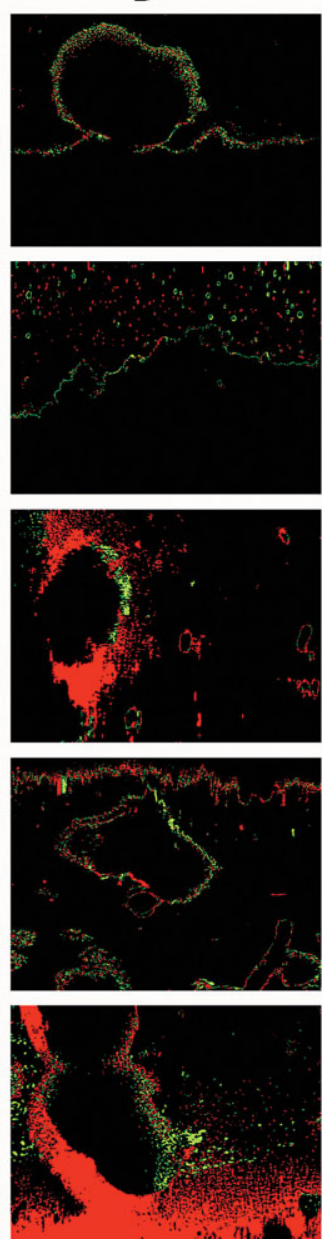

Figure 3. Visualization of biofilm removal and shape changes in control, chlorhexidine, DispersinB, iron chloride, and urea treatment sequences. In Columns B-D, thresholded binary images were subtracted to visualize the change in biomass structure during a particular interval. Red indicates pixels previously containing biomass that became empty, green indicates pixels previously empty that became filled. Column $A$, transmission image prior to flow challenge; Column $B$, change resulting from pre-treatment flow challenge; Column $C$, change during treatment soak period; Column $D$, change resulting from post-treatment flow challenge. Scale bar, $200 \mu \mathrm{m}$.

each of the three phases of the experiment. In Columns B and $\mathrm{C}$ of the chlorhexidine experiment, the mix of red and green indicates that the biofilm was reshaped somewhat during the pre-treatment flow challenge and treatment soak phases of the experiment. In Column D of the chlorhexidine treatment, the near absence of color indicates that the biofilm was scarcely perturbed by the post-treatment flow challenge. The predominance of red in Columns $C$ and D of the DispersinB treatment suggests that the enzyme causes net removal of biofilm in the soak and post-treatment flow challenge phases of the experiment. In the iron chloride experiment, the cell cluster under observation deforms in the flow direction during the pre-treatment flow challenge and does not completely recover its original position. This is evident in the band of red color along the upstream edge of the cluster and green color along the downstream edge of the cluster (Column B). During the soak in iron chloride solution, the cluster contracts slightly, yielding a red ring around the biofilm in Column C. The distinction between contraction and removal cannot be inferred from Figure 3 but is evident in watching video of these experimental sequences. S. epidermidis biofilms treated with urea swell. This is apparent in the large green halo in Column C (treatment soak) of the urea experiment. During the subsequent post-treatment flow challenge, substantial biomass is lost leading to predominantly red color in Column D.

The change in biomass area seen in Figure 3 was quantified during the different phases of the creep test and the results are included in Table II. A change in biomass area is shown for the pre-treatment test, the post-treatment test and loss during the 15-min treatment, with a negative percentage implying that the biofilm area increased. For the untreated control, changes in area were less than an average of $2.3 \%$ throughout the test. However, significant swelling during the treatment phase was seen in both the 
Table II. Percent biomass area losses during $15 \mathrm{~s}$ pre and post-treatment fluid shear and during treatment soak. Negative numbers correspond to increases in area biofilm area.

\begin{tabular}{|c|c|c|c|c|}
\hline & & $\begin{array}{l}\% \text { Loss during } \\
\text { pre-treatment }\end{array}$ & $\begin{array}{l}\% \text { Loss during } \\
\text { post-treatment }\end{array}$ & $\begin{array}{l}\text { \% Loss during } \\
\text { treatment }\end{array}$ \\
\hline \multirow[t]{4}{*}{ Control treatment } & Trial \#1 & 1.0 & 2.7 & -2.3 \\
\hline & Trial \#2 & -0.9 & 0.5 & -3.1 \\
\hline & Trial \#3 & 6.9 & -0.1 & 0.9 \\
\hline & Average $\pm \mathrm{SD}$ & $2.3 \pm 4.1$ & $1.0 \pm 1.5$ & $-1.5 \pm 2.1$ \\
\hline \multirow[t]{4}{*}{ Iron chloride treatment } & Trial \#1 & 7.4 & 2.7 & 8.9 \\
\hline & Trial \#2 & 1.3 & 10.9 & 9.6 \\
\hline & Trial \#3 & 6.1 & -0.1 & 6.4 \\
\hline & Average $\pm S D$ & $4.9 \pm 3.2$ & $4.4 \pm 5.7$ & $8.3 \pm 1.7$ \\
\hline \multirow[t]{4}{*}{ Chlorhexidine treatment } & Trial \#1 & -3.1 & 0.4 & 14.0 \\
\hline & Trial \#2 & -0.2 & 0.5 & -6.4 \\
\hline & Trial \#3 & -0.6 & 6.0 & -70.8 \\
\hline & Average \pm SD & $-1.3 \pm 1.6$ & $2.3 \pm 3.2$ & $-21.1 \pm 44$ \\
\hline \multirow[t]{4}{*}{ Urea treatment } & Trial \#1 & 1.3 & 70.7 & -58.8 \\
\hline & Trial \#2 & -0.5 & 62.0 & -48.1 \\
\hline & Trial \#3 & 14.4 & 81.4 & -53.5 \\
\hline & Average $\pm \mathrm{SD}$ & $5.1 \pm 8.1$ & $71.4 \pm 9.7$ & $-53.4 \pm 5.3$ \\
\hline \multirow[t]{4}{*}{ DispersinB treatment } & Trial \#1 & -0.4 & 24.3 & 14.2 \\
\hline & Trial \#2 & -10.0 & 56.2 & 28.5 \\
\hline & Trial \#3 & -4.9 & 38.9 & 25.5 \\
\hline & Average \pm SD & $-5.1 \pm 4.7$ & $39.8 \pm 15$ & $22.7 \pm 7.5$ \\
\hline
\end{tabular}

chlorhexidine (21\%) and urea treatments (53\%). Also, substantial biofilm removal was seen in the post-treatment tests of urea $(71 \%)$ and DispersinB $(40 \%)$. It is important to note that the extent of post-treatment deformation correlates with biofilm removal for treatments that increased deformation and treatments that decreased deformation. Treatments that caused a large increase in post-treatment deformation (urea, DispersinB) resulted in statistically significantly greater removal $(P=0.002)$ compared to treatments that resulted in $<20 \%$ increase in post-treatment deformation (iron chloride, chlorhexidine).

Whereas the repeated control did not demonstrate significant change in deformation, all four treatments changed the mechanical behavior and failure of the biofilm. Treatment with iron chloride caused the biofilm to deform less (Fig. 2B and Table I) in response to the hydrodynamic force. The biofilm appeared to contract slightly (8\% reduction in area) during the iron chloride soak, Table II, and large-scale failure of the biofilm is not evident. Similar behavior was observed when biofilms were treated with chlorhexidine. The biofilm expanded during the antimicrobial soak period ( $21 \%$ expansion in area, Table II). The chlorhexidine-treated biofilm had a smaller maximum deformation in response to the creep test (Fig. 2C and Table I).

Treating biofilms with urea and DispersinB caused the biofilm to have higher deformations in response to the creep test (Fig. 2D, Fig. 2E, and Table I). Treatment with urea resulted in swelling of the biofilm during the treatment soak period as shown in Table II. Extensive biofilm removal (71\% reduction in area) was observed in the urea-treated biofilm when challenged by the creep test. The DispersinB treatment also resulted in removal of biofilm, especially from the periphery of treated cell clusters (40\% reduction in area), and recapitulated in the number of detachments and failures seen in Table I.

\section{Micro Indentation Results}

Indentation results of the biofilm in the untreated, or control, condition at different loading rates are shown in Figure 4. These results show that higher loading rates result in higher forces as the indenter is extended into the biofilm, indicative of a viscous material response. Also seen is a linear slope in the unloading portion of the test, indicating an elastic response is also present within the material. The hysteresis in the curve represents dissipated energy within the deformation process.

Panel B in Figure 4 shows the force-displacement curve at an identical location on the biofilm before and after treatment with DispersinB, and is representative of the data seen for each treatment. The maximum force for both the pre-treatment test and the post-treatment test were recorded. Table III shows the post-treatment to pretreatment ratio of maximum force for all three trials and loading rates. The control for this procedure consisted of repeating an indent at the identical location without treating the biofilm and showed an $11 \%$ drop in maximum force. The average maximum force for both the urea and the DispersinB is less than one, indicating that these treatments have a smaller post-treatment maximum force, and are thus more compliant. The opposite is true, qualitatively, for both the iron chloride and chlorhexidine, in that these agents become stiffer after the treatment. Notice that chlorhexidine has an average maximum force value twice that of iron 

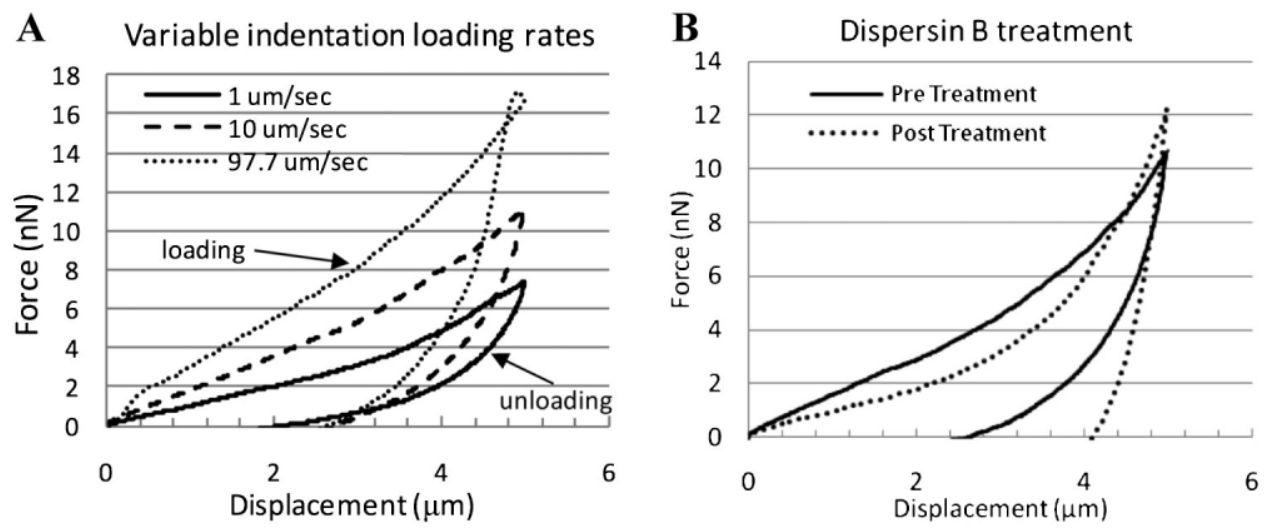

Figure 4. Force displacement curves for (A) variable loading rates on $S$. epidermidis biofilm (B) $10 \mu \mathrm{m} / \mathrm{s}$ loading rate indentation tests after DispersinB treatment.

chloride, implying that chlorhexidine is a stronger stiffening agent than the iron chloride.

\section{Discussion}

The two main objectives in this paper were to develop and apply techniques to characterize the mechanical deformation and failure nature of $S$. epidermidis biofilms and demonstrate change in biofilm material properties in response to chemical and enzymatic treatments. The first technique was a hydrodynamic shear methodology in which S. epidermidis biofilm was grown in a capillary flow cell. The biofilm was subjected to a hydrodynamic stress through fluid flow for fifteen seconds, while deformations were

Table III. Maximum force of each trial with post-treatment maximum displacement divided by pre-treatment maximum displacement.

\begin{tabular}{|c|c|c|}
\hline & & $\begin{array}{c}\text { Max force } \\
\text { (post-treatment/pre-treatment) }\end{array}$ \\
\hline \multirow{4}{*}{ Control treatment } & Trial \#1 & 1.02 \\
\hline & Trial \#2 & 0.85 \\
\hline & Trial \#3 & 0.79 \\
\hline & Average $\pm \mathrm{SD}$ & $0.89 \pm 0.12$ \\
\hline \multirow[t]{4}{*}{ Iron chloride treatment } & Trial \#1 & 1.22 \\
\hline & Trial \#2 & 1.14 \\
\hline & Trial \#3 & 0.84 \\
\hline & Average $\pm \mathrm{SD}$ & $1.06 \pm 0.20$ \\
\hline \multirow[t]{4}{*}{ Chlorhexidine treatment } & Trial \#1 & 1.90 \\
\hline & Trial \#2 & 3.29 \\
\hline & Trial \#3 & 1.33 \\
\hline & Average $\pm \mathrm{SD}$ & $2.17 \pm 1.01$ \\
\hline \multirow[t]{4}{*}{ Urea treatment } & Trial \#1 & 0.41 \\
\hline & Trial \#2 & 0.63 \\
\hline & Trial \#3 & 1.07 \\
\hline & Average $\pm \mathrm{SD}$ & $0.70 \pm 0.34$ \\
\hline \multirow{4}{*}{ DispersinB treatment } & Trial \#1 & 0.31 \\
\hline & Trial \#2 & 0.72 \\
\hline & Trial \#3 & 1.09 \\
\hline & Average $\pm \mathrm{SD}$ & $0.70 \pm 0.39$ \\
\hline
\end{tabular}

optically recorded. The biofilm was treated with either a chemical or enzymatic agent, after which another hydrodynamic stress was applied.

Creep curves like those reported in Figure 2 do not allow facile determination of intrinsic material properties parameters such yield strength or visco-elastic moduli (Aggarwal et al., 2010). A careful 3-D reconstruction of the biofilm cluster shape, coupled to fluid dynamic modeling would be necessary to perform this analysis. There have been several modeling studies that move towards solving this computationally challenging problem (Alpkvist and Klapper 2007; Picioreanu et al., 2001; Taherzadeh et al., 2010; Towler et al., 2007; Vo et al., 2010). Nevertheless, the change in the creep curve resulting from a chemical or enzymatic treatment provides an integrated quantitative description of the degree to which the mechanical properties of the biofilm structure have been affected.

The capillary flow cell is a powerful technique in visualizing the effect of treatments on the biofilm structure. Additionally, failure events and removal of the biofilm can be observed through this technique. The limitation of this technique in gathering quantitative displacement data is seen in the large variations in Table I, which is due in part to the 3-D fluid-structure interaction (Vo and Heys, 2011; Vo et al., 2010). The inherent variation in biofilm mechanical response, as identified in the literature (Aggarwal and Hozalski, 2010; Cense et al., 2006; Lau et al., 2009a, 2009b; Stoodley et al., 2002), would also contribute to the scatter seen in the results of Table I. Statistical analysis of the maximum displacements in Table I show that only the urea treatment is statistically different, at $10 \%$ significance, from the control. This analysis strengthens the argument that biofilm material properties are inherently varied and difficult to measure, while making definitive material properties difficult to confirm; however, taken as a complete experiment including material deformation, material failures, and biofilm area, the influence of iron chloride, chlorhexidine, urea, and DispersinB on the mechanical 

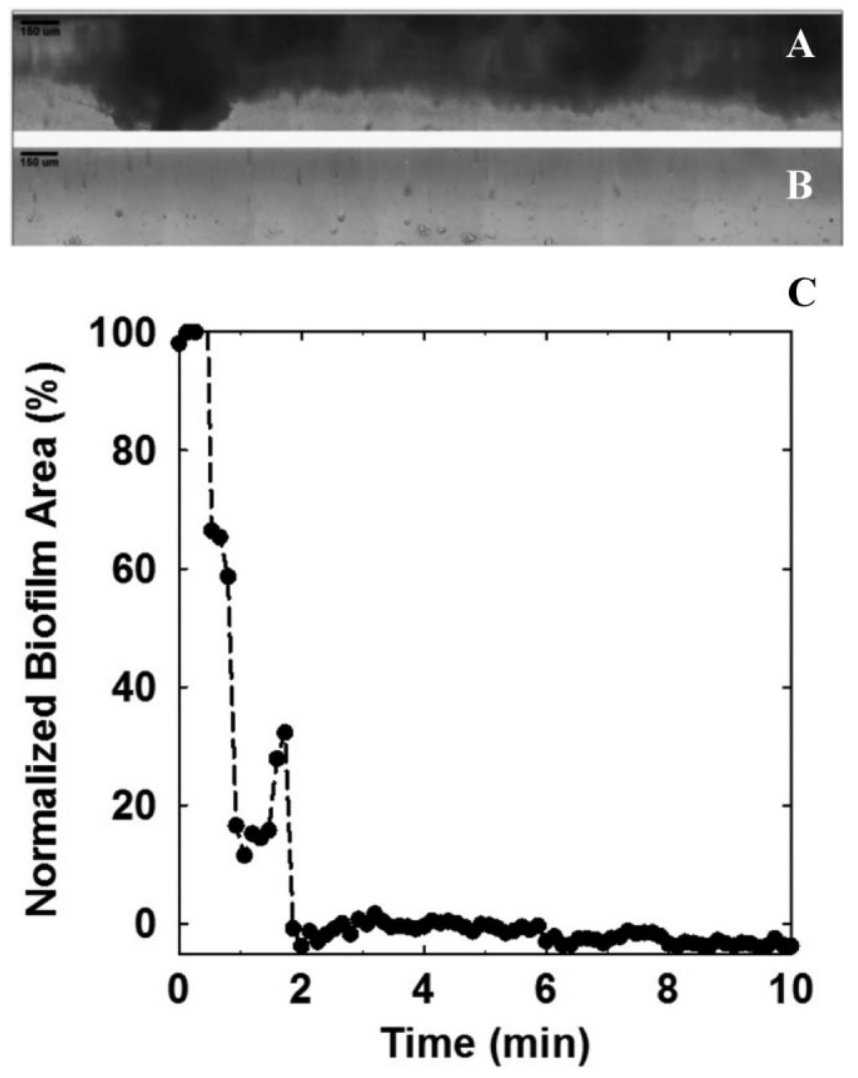

Figure 5. S. epidermidis biofilm imaged in a capillary flow cell reactor before $(\mathbf{A})$ and after $(\mathbf{B}) 1 \mathrm{~h}$ treatment with $6.0 \mathrm{~g} / \mathrm{L}(0.1 \mathrm{M})$ urea delivered continuously at a flow rate of $10 \mathrm{~mL} / \mathrm{min}$ (10 times the flow rate at which the biofilm was grown). Most of the biofilm removal occurred in the first few minutes (C).

response and failure of the $S$. epidermidis has been characterized by the flow cell technique. A correlation has also been established that for biofilm removal coupled to hydrodynamic forces, an increase in deformability can result in increased biofilm failure.

The protocol used in this investigation of a 15 min static soak with a treatment agent followed by a $15 \mathrm{~s}$ fluid shear challenge was designed to collect data on how biofilm mechanical integrity is altered by the treatment. This protocol does not optimize biofilm removal. To illustrate the potential for treatment agents in concert with mechanical challenge to achieve progressive biofilm removal, we subjected a $S$. epidermidis biofilm to a continuously flowing solution of $6.0 \mathrm{~g} / \mathrm{L}(0.1 \mathrm{M})$ urea (Fig. 5). Within $3 \mathrm{~min}$, a significant portion of the biofilm was removed. This simple experiment illustrates that treatments that reduce biofilm cohesion, when applied in conjunction with a mechanical challenge, can achieve substantial biofilm removal.

The second technique utilized an AFM to mechanically interrogate the biofilm by indenting a tip of known geometry into the biofilm surface. S. epidermidis biofilm was grown on silicon wafers which were placed under the
AFM indenter. The biofilm was indented to a depth of $5 \mu \mathrm{m}$, treated, and then indented to a depth of $5 \mu \mathrm{m}$ at the same location. The indentation technique interrogates the biofilm in a compressive loading state, and is therefore unable to study the failure mechanisms of the biofilm. However, the effect of the treatment on the deformation properties of biofilm has been supported by indentation tests. The major limitation to this technique was the variability in biofilm thickness and geometry requiring measurements to be made at identical points on the biofilm structure to compare pretreatment and post-treatment response.

The capillary flow cell and indentation experiments on S. epidermidis biofilm collectively suggest the following interpretations: (1) chemical or enzymatic treatments, including those that are not frankly antimicrobial, can alter the cohesion of bacterial biofilm; (2) biocidal treatments (e.g., chlorhexidine) do not necessarily weaken the biofilm; and (3) biofilm removal following treatment with agents that make the biofilm more deformable (e.g., urea, DispersinB) depend on interaction between the moving fluid and the biofilm structure. Measurements such as those reported here open the door to development of new technologies for controlling detrimental biofilms by targeting biofilm cohesion rather than killing microorganisms.

The author's acknowledge the assistance of Betsey Pitts with image preparation and Al Parker for statistical analysis. This work was supported by NSF Award Number: 0728621.

\section{References}

Aggarwal S, Hozalski RM. 2010. Determination of biofilm mechanical properties from tensile tests performed using a micro-cantilever method. Biofouling 26(4):479-486.

Aggarwal S, Poppele EH, Hozalski RM. 2010. Development and testing of a novel microcantilever technique for measuring the cohesive strength of intact biofilms. Biotechnol Bioeng 105(5):924-934.

Ahimou F, Semmens MJ, Novak PJ, Haugstad G. 2007. Biofilm cohesiveness measurement using a novel atomic force microscopy methodology. Appl Environ Microbiol 73:2897-2904.

Alpkvist E, Klapper I. 2007. Description of mechanical response including detachment using a novel particle model of biofilm/flow interaction. Water Sci Technol 55(8-9):265-273.

Cense AW, Peeters EAG, Gottenbos B, Baaijem FPT, Nuijs AM, van Dongen MEH. 2006. Mechanical properties and failure of Streptococcus mutans biofilms, studied using a microindentation device. J Microbiol Methods 67(3):463-472.

Chaignon P, Sadovskaya I, Ragunah C, Ramasubbu N, Kaplan JB, Jabbouri S. 2007. Susceptibility of staphylococcal biofilms to enzymatic treatments depends on their chemical composition. Appl Microbiol Biotechnol 75(1):125-132.

Chen MJ, Zhang Z, Bott TR. 2005. Effects of operating conditions on the adhesive strength of Pseudomonas fluorescens biofilms in tubes. Colloid Surface B 43(2):61-71.

Chen X, Stewart PS. 2000. Biofilm removal caused by chemical treatments. Water Res 34(17):4229-4233.

Chen X, Stewart PS. 2002. Role of electrostatic interactions in cohesion of bacterial biofilms. Appl Microbiol Biotechnol 59(6):718-720.

Costerton JW, Stewart PS, Greenberg EP. 1999. Bacterial biofilms: A common cause of persistent infections. Science 284(5418):1318-1322.

Davison WM, Pitts B, Stewart PS. 2010. Spatial and temporal patterns of biocide action against Staphylococcus epidermidis biofilms. Antimicrob Agents Chemother 54(7):2920-2927. 
Flemming H, Wingender J, Mayer C, Korstgens V, Borchard W. editors. 2000. Cohesiveness in biofilm matrix systems. New York, NY: Cambridge University Press. p. 87-105.

Jones WL, Sutton MP, McKittrick L, Stewart PS. 2011. Chemical and antimicrobial treatments change the viscoelastic properties of bacterial biofilms. Biofouling 27(2):207-215.

Kaplan JB, Ragunath C, Ramasubbu N, Fine DH. 2003. Detachment of Actinobacillus actinomycetemcomitans biofilm cells by an endogenous beta-hexosaminidase activity. J Bacteriol 185(16):4693-4698.

Lau PCY, Dutcher JR, Beveridge TJ, Lam JS. 2009a. Absolute quantitation of bacterial biofilm adhesion and viscoelasticity by microbead force spectroscopy. Biophys J 96(7):2935-2948.

Lau PCY, Lindhout T, Beveridge TJ, Dutcher JR, Lam JS. 2009b. Differential lipopolysaccharide core capping leads to quantitative and correlated modifications of mechanical and structural properties in Pseudomonas aeruginosa biofilms. J Bacteriol 191:(21).

Lieleg O, Caldara M, Baumgärtel R, Ribbeck K. 2011. Mechanical robustness of Pseudomonas aeruginosa biofilms. Soft Matter 7(7):3307-3314.

Mohle RB, Langemann T, Haesner M, Augustin W, Scholl S, Neu TR, Hempel DC, Horn H. 2007. Structure and shear strength of microbial biofilms as determined with confocal laser scanning microscopy and fluid dynamic gauging using a novel rotating disc biofilm reactor. Biotechnol Bioeng 98:747-755.

Ohashi A, Harada H. 1994. Adhesion strength of biofilm developed in an attached-growth reactor. Water Sci Technol 29(10-11):281-288.

Ohashi A, Harada H. 1996. A novel concept for evaluation of biofilm adhesion strength by applying tensile force and shear force. Water Sci Technol 34(5-6):201-211.

Ohashi A, Koyama T, Syutsubo K, Harada H. 1999. A novel method for evaluation of biofilm tensile strength resisting erosion. Water Sci Technol 39(7):261-268.

Picioreanu C, van Loosdrecht MCM, Heijnen JJ. 2001. Two-dimensional model of biofilm detachment caused by internal stress from liquid flow. Biotechnol Bioeng 72(2):205-218.

Poppele EH, Hozalski RM. 2003. Micro-cantilever method for measuring the tensile strength of biofilms and microbial flocs. J Microbiol Methods 55(3):607-615.

Simoes M, Pereira MO, Vieira MJ. 2005a. Action of a cationic surfactant on the activity and removal of bacterial biofilms formed under different flow regimes. Water Res 39(2-3):478-486.

Simoes M, Pereira MO, Vieira MJ. 2005b. Effect of mechanical stress on biofilms challenged by different chemicals. Water Res 39(20):51425152.
Simoes M, Simoes LC, Vieira MJ. 2009. Species association increases biofilm resistance to chemical and mechanical treatments. Water Res 43(1): 229-237.

Stoodley P, Cargo R, Rupp CJ, Wilson S, Klapper I. 2002. Biofilm material properties as related to shear-induced deformation and detachment phenomena. J Ind Microbiol Biotechnol 29(6):361-367.

Stoodley P, Jacobsen A, Dunsmore BC, Purevdorj B, Wilson S, Lappin-Scott HM, Costerton JW. 2001. The influence of fluid shear and $\mathrm{AlCl}_{3}$ on the material properties of Pseudomonas aeruginosa PAO1 and Desulfovibrio sp. EX265 biofilms. Water Sci Technol 43(6):113-120.

Stoodley P, Lewandowski Z, Boyle JD, Lappin-Scott HM. 1999. Structural deformation of bacterial biofilms caused by short-term fluctuations in fluid shear: An in situ investigation of biofilm rheology. Biotechnol Bioeng 65(1):83-92.

Taherzadeh D, Picioreanu C, Kuttler U, Simone A, Wall WA, Horn H. 2010. Computational study of the drag and oscillatory movement of biofilm streamers in fast flows. Biotechnol Bioeng 105(3):600610.

Towler BW, Cunningham A, Stoodley P, McKittrick L. 2007. A model of fluid-biofilm interaction using a Burger material law. Biotechnol Bioeng 96(2):259-271.

Townsin RL. 2003. The ship hull fouling penalty. Biofouling 19:9-15.

Vieira M, Melo L, Pinheiro M. 1993. Biofilm formation: Hydrodynamic effects on internal diffusion and structure. Biofouling 7(1):67-80.

Vinogradov A, Winston M, Rupp C, Stoodley P. 2004. Rheology of biofilms formed from the dental plaque pathogen Streptococcus mutans. Biofilms 1:49-56.

Vo GD, Brindle E, Heys J. 2010. An experimentally validated immersed boundary model of fluid-biofilm interaction. Water Sci Technol 61(12):3033-3040.

Vo GD, Heys J. 2011. Biofilm deformation in response to fluid flow in capillaries. Biotechnol Bioeng 108(8):1893-1899.

Walker JT, Mackerness CW, Mallon D, Makin T, Williets T, Keevil CW. 1995. Control of Legionella-pneumophila in a hospital water-system by chlorine dioxide. J Industrial Microbiol 15(4):384-390.

Wloka M, Rehage H, Flemming HC, Wingender J. 2004. Rheological properties of viscoelastic biofilm extracellular polymeric substances and comparison to the behavior of calcium alginate gels. Colloid Polym Sci 282(10):1067-1076.

Xavier JB, Picioreanu C, Rani SA, van Loosdrecht MCM, Stewart PS. 2005. Biofilm-control strategies based on enzymic disruption of the extracellular polymeric substance matrix - A modelling study. MicrobiolSGM 151:3817-3832. 